\title{
MODELLING THE RESPONSE OF VASCULAR TUMOURS TO CHEMOTHERAPY: A MULTISCALE APPROACH
}

\author{
HELEN M. BYRNE* and MARKUS R. OWEN ${ }^{\dagger}$ \\ Centre for Mathematical Medicine, School of Mathematical Sciences, \\ University of Nottingham, Nottingham NG7 2RD, England \\ *helen.byrne@nottingham.ac.uk \\ ${ }^{\dagger}$ markus.owen@nottingham.ac.uk \\ TOMAS ALARCON \\ Bioinformatics Unit, Department of Computer Science \\ University College London, Gower Street, London WC1E 6BT, England \\ t.alarcon@cs.ucl.ac.uk \\ JAMES MURPHY \\ Centre for Mathematical Medicine, School of Mathematical Sciences \\ University of Nottingham, Nottingham NG7 2RD, England \\ PHILIP K. MAINI \\ Centre for Mathematical Biology, Mathematical Institute, \\ University of Oxford, 24-29 St Giles, Oxford OX1 SLB, England \\ maini@maths.ox.ac.uk
}

Received 31 October 2005

Revised 6 December 2005

Communicated by N. Bellomo

\begin{abstract}
An existing multiscale model is extended to study the response of a vascularised tumour to treatment with chemotherapeutic drugs which target proliferating cells. The underlying hybrid cellular automaton model couples tissue-level processes (e.g. blood flow, vascular adaptation, oxygen and drug transport) with cellular and subcellular phenomena (e.g. competition for space, progress through the cell cycle, natural cell death and drug-induced cell kill and the expression of angiogenic factors). New simulations suggest that, in the absence of therapy, vascular adaptation induced by angiogenic factors can stimulate spatio-temporal oscillations in the tumour's composition.

Numerical simulations are presented and show that, depending on the choice of model parameters, when a drug which kills proliferating cells is continuously infused through the vasculature, three cases may arise: the tumour is eliminated by the drug; the tumour continues to expand into the normal tissue; or, the tumour undergoes spatiotemporal oscillations, with regions of high vascular and tumour cell density alternating
\end{abstract}


with regions of low vascular and tumour cell density. The implications of these results and possible directions for future research are also discussed.

Keywords: Multiscale modelling; hybrid cellular automaton; cancer; chemotherapy.

AMS Subject Classification: 92C35, 92C37, 93A30

\section{Introduction}

Even with widespread access to (and improvements in) screening techniques and the development of more effective treatments, cancer remains a major cause of premature morbidity in the Western world. While cure rates have improved dramatically for some tumours (e.g. breast and colorectal cancers), ${ }^{20}$ patient prognosis is not consistently good across all cancers (e.g. lung cancer). The reason for this remains an open question: is it due to increasing life expectancy, the cumulative effect of exposure to carcinogenic compounds, diet or simply more accurate diagnosis resulting from better screening? What is clear, though, is that the vast resources being invested in cancer biology and drug discovery have not yet translated into significant improvements in the treatment and eradication of the disease. In order to make further progress, clinicians need to understand why one cancer patient responds well to treatment while another, similar patient does not. A key issue here is the scheduling of therapy. In particular, if combinations of drugs are to be used, how should they be coordinated? What level of patient-specific information is needed to ensure optimisation of individual-based treatment protocols?

We contend that mathematics provides a natural framework within which such questions can be studied. Since results can be obtained on a shorter timescale than the corresponding experimental analyses, a wider range of alternatives can be explored. ${ }^{25}$ Even so, we do not believe that mathematical modelling and simulation will ever replace experimental work. Rather, it will form part of the toolkit available to biologists, being used in tandem with experimental work, to suggest the most promising directions for future research and, given sufficiently accurate data, to predict those patients who are likely to respond to a particular drug or treatment protocol.

Increasing numbers of theoreticians are now constructing mathematical models that can be used to predict how the different physical mechanisms that act within a tumour combine to produce observed phenomena and to determine the response of specific tumours to chemotherapy (see Refs. 8, 38, 45 and 48 for reviews). For example, early models developed by Greenspan and others ${ }^{1,28,29,37}$ to describe the development of multicellular tumour spheroids (MTS) cultured in vitro assume that growth is regulated by a single, diffusible nutrient (typically oxygen or glucose). In spite of their simplicity, such models reproduce the dynamics of MTS and their characteristic spatial structure, with fully-developed MTS comprising an outer rim of nutrient-rich, rapidly proliferating cells, a central core of nutrient-deficient or necrotic cellular debris and an intermediate annular region containing quiescent or non-proliferating cells. ${ }^{16,30,31}$ These spatially-structured models have been extended to predict the response of early (avascular) and mature (vascularised) tumours to 
chemotherapy. ${ }^{33-35}$ Other authors have developed lumped-parameter models, formulated as systems of ordinary differential equations (ODEs) to study the response of specific tumours growing in vivo to treatment ${ }^{7,15,42}$ and age-structured models to predict the response of different tumour cell lines to drugs that target specific phases of the cell cycle. ${ }^{9}$

The variety of factors involved in the development of solid tumours stems, to a large extent, from the fact that "cancer" is a generic term, used to characterize a spectrum of disorders that share common features. At this generic level of description, cancer may be viewed as a cellular disease in which controls that usually regulate growth and maintain homeostasis are disrupted. Cancer is typically initiated by genetic mutations that lead to enhanced proliferation rates (or reduced rates of apoptosis) and the formation of an avascular tumour. Since it receives nutrients by diffusion, the size of an avascular tumour is limited to several millimeters in diameter. Further growth relies on the tumour producing growth factors, including vascular endothelial growth factor (VEGF), that stimulate the ingrowth of a new blood supply from the host vasculature via angiogenesis. ${ }^{17,18}$ Once vascularised, the tumour has access to a vast nutrient source and rapid growth ensues. Further, clusters of tumour cells that break away from the primary tumour, on entering the vasculature, may be transported to other organs where they establish secondary tumours or metastases that further compromise the host. Invasion is another key feature of solid tumours whereby contact with the host tissue stimulates the production of enzymes that digest it, liberating space into which the tumour cells migrate and/or proliferate. Thus, cancer is a complex, multiscale process. The spatial scales of interest range from the subcellular level, to the cellular and macroscopic (or tissue) levels while the timescales may vary from seconds (or less) for signal transduction pathways to months for tumour doubling times.

In an attempt to account for its multiscale nature, Alarcón and co-workers ${ }^{4,5}$ have formulated a hybrid cellular automaton of vascular tumour growth that extends earlier work by Gatenby and co-workers ${ }^{43}$ and links submodels which describe processes operating on different spatial scales. Progress through the cell cycle and the production of VEGF are incorporated at the subcellular level while cell-cell communication and competition for resources are considered at the cellular level. Finally, transport of nutrients and VEGF, blood flow and vascular adaptation are included at the macro- or tissue scale (see Fig. 1). Coupling between the different submodels is achieved in several ways. For example, local oxygen levels which are determined at the macroscale influence both progress through the cell cycle and VEGF production at the subcellular level. Conversely, the intracellular production of VEGF modulates vascular adaptation at the macroscale.

While the model of vascular tumour growth described above is undoubtedly complex, it has the potential to yield many predictions that could stimulate new experimental investigations. One natural avenue that we explore here involves using the model to compare the response of vascular tumours to standard therapies that kill proliferating cells (e.g. doxorubicin and paclitaxel ${ }^{33,42}$ ). Equally, we could investigate the response to antiangiogenic compounds (e.g. angiostatin, 


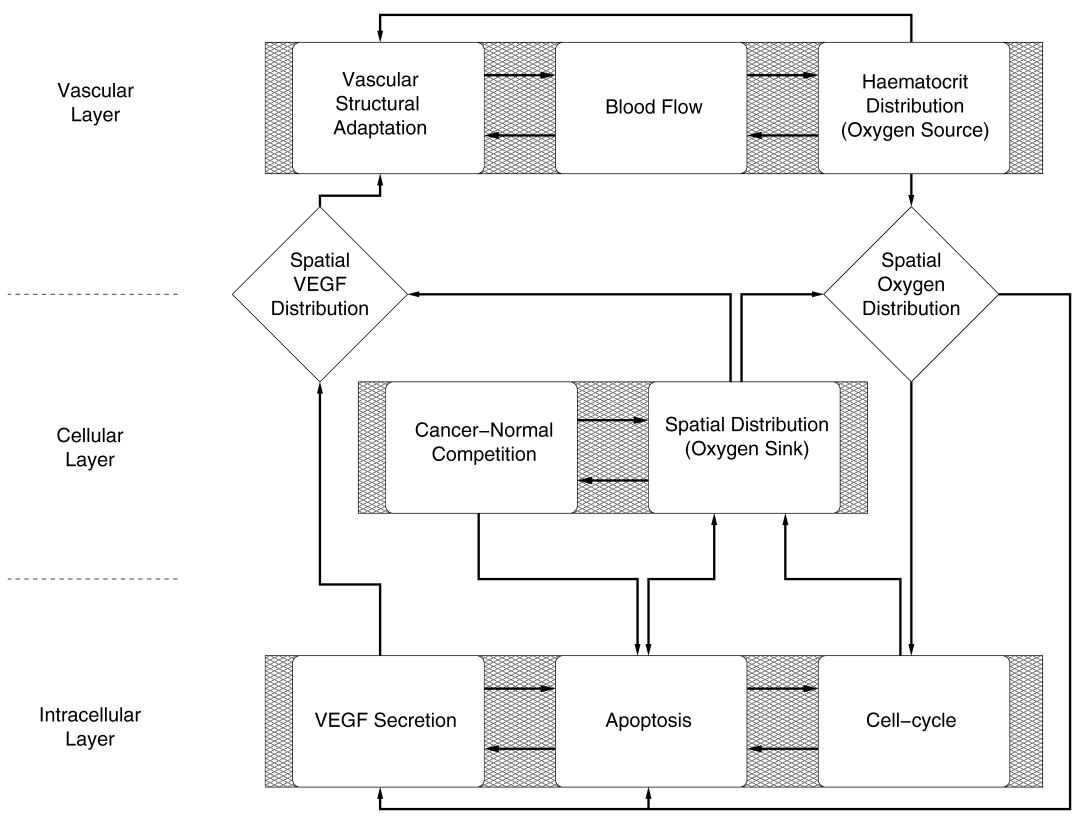

Fig. 1. Schematic diagram illustrating the structure of our hybrid cellular automaton model.

combretastain and endostatin ${ }^{19,40,53}$ ) that target the tumour vasculature. Our modelling approach shares several common features with complementary work by Anderson and coworkers. ${ }^{36,51}$ Like them, we base our submodel of vascular adaptation and blood flow on work by Pries et al. ${ }^{46}$ and view the vessels as distributed sources for blood-borne nutrients and drugs. However, Anderson and co-workers focus their simulations on a relatively short timescale following a bolus injection and consider how the vascular network influences the predicted distribution of the drug within the tissue. By contrast, we couple blood flow and the tissue dynamics and consider longer timescales over which effects associated with the drug's mode of action are discernible. In consequence, we are able to obtain detailed spatiotemporal information about the level of tumour cell kill and the extent of damage to healthy tissue in response to different anticancer therapies. For example, when using the model to predict the tumour's response to drugs that target proliferating cells, we observe three types of behaviour: the drug is ineffective and the tumour continues to grow; the drug is effective and the tumour is eliminated; or, spatio-temporal oscillations are observed in which well-oxygenated (and drug-rich) regions of high vascular and tumour cell density alternate over time with regions of widespread cell death and reduced blood flow.

The remainder of the paper is organised as follows. In Sec. 2 we introduce our hybrid model of vascular tumour growth. In Sec. 3 we extend the model to investigate the response of a vascular tumour to treatment with an antiproliferative chemotherapeutic agent and discuss the different outcomes that can arise. Finally, 
in Sec. 4 we summarise and interpret our results and discuss possible directions for future research. We also discuss the mathematical challenges raised by this modelling approach.

\section{Model Development}

Our multiscale model describes the growth of a tumour located within a region of normal, vascularised tissue. It accounts for a variety of inter-related phenomena which operate on different space and time scales. These features include cell division, the expression of angiogenic factors (e.g. VEGF), competition between normal and cancer cells, blood flow, oxygen transport and vascular adaptation. The model is formulated as a hybrid cellular automata, with different submodels describing behaviour at the subcellular, cellular and macroscopic (or vascular) levels (see Fig. 1). Since the focus of this paper is on the tumour's response to chemotherapy, we summarise each submodel below and refer the reader to Refs. 4 and 5 for further details. We stress that the submodels we describe below simply illustrate how such a multiscale model can be assembled: the framework we present is general, with considerable scope for incorporating more realistic (and, hence, complex) submodels. This raises an important issue, namely whether the level of information and detail incorporated at each spatial scale influences the system's behaviour: this will form the basis of future research. ${ }^{10}$

\subsection{The vascular network (macroscale)}

We consider for simplicity a vascular structure which is composed of a regular hexagonal network (similar to that in liver tissue). We impose a pressure drop across the vasculature, assuming that blood flows into the idealised "tissue" through a single inlet vessel and drains through a single outlet vessel. To compute the blood flow through each vessel we use the Poiseuille approximation, and, given the initial network configuration (i.e. radii and lengths) we compute the flow rates through, and pressure drops across, each vessel using Kirchhoff's laws. We assume that initially all vessels have the same radius, but allow them to undergo structural adaptation over time. Following Pries et $a l .{ }^{46}$ we assume that the radius $R(t)$ of a vessel evolves over a time period $\Delta t$ as follows:

$$
R(t+\Delta t)
$$


In Eq. (2.1) $\dot{Q}$ is the flow rate, $\dot{Q}_{\text {ref }}, k_{m}$ and $k_{s}$ are constants, $H$ is the haematocrit (red blood cell volume), $\tau_{w}=R \Delta \pi / L$ is the wall shear stress acting on a vessel of length $L$ and $\pi$ is the transmural pressure. The magnitude of the corresponding "set point" value of the wall shear stress, $\tau(\pi)$, is obtained from an empirical fit to experimental data. Thus the second term on the right-hand side of Eq. (2.1) represents the response to haemodynamic stimuli. The third term describes the metabolic stimulus which we assume increases as the haematocrit decreases. Coupling to the subcellular level is achieved by assuming that $k_{m}=k_{m}(V)$ where $V$ denotes the local, extracellular VEGF concentration (see Eq. (2.3) below). We account for the pro-angiogenic impact of VEGF by assuming that $k_{m}(V)$ is an increasing, saturating function, of the form:

$$
k_{m}(V)=k_{m}^{0}\left(1+k_{m}^{V} \frac{V}{V_{0}+V}\right),
$$

for constants $k_{m}^{0}, k_{m}^{V}$ and $V_{0}$. Finally, the constant $k_{s}$ represents the so-called shrinking tendency and is included so that, in the absence of mechanical and metabolic stimuli, the vessel will atrophy. Pries et al. found that a third stimulus was required for efficient structural adaptation. ${ }^{46}$ We justify omitting this phenomenon from our model on the basis that tumour vasculature adapts less efficiently than normal vasculature.

Once Eq. (2.1) has been used to update each vessel radius, the associated blood flow and haematocrit can be determined. When updating the blood flow, we use a complex function of $H$ and $R$ which was determined empirically in Ref. 46 to describe blood viscosity. When updating the haematocrit, we assume that at branch points $H$ splits in a manner which is proportional to the flow velocity in each daughter vessel. ${ }^{21}$

\subsection{The diffusible chemicals (macroscale)}

Coupling between the vascular, cellular and subcellular scales is effected by the diffusive transport of oxygen and VEGF, under the assumption that oxygen is the single, growth-rate limiting nutrient. We calculate their respective distributions within the tissue by solving appropriate reaction-diffusion equations and imposing zero-flux boundary conditions. We justify adopting the usual quasi-steady approximation in these equations on the grounds that the timescales for oxygen and VEGF diffusion are much shorter than the tumour doubling time which is the timescale of interest (minutes and weeks or months, respectively).

We denote by $P$ the oxygen concentration and treat the cells as sinks and the vessels as sources of oxygen so that the relevant diffusion equation can be written:

$$
0=D_{P} \nabla^{2} P+h_{P}\left(P_{\text {vess }}-P\right)-\lambda_{\text {cell }} P .
$$


In (2.2), $D_{P}$ denotes the assumed constant oxygen diffusion coefficient, $h_{P}$ is the rate at which oxygen is transported across the vessel wall $\left(h_{P}\right.$ is only nonzero where vessels are located), $P_{\text {vess }}=P_{\text {vess }}(H)$ is the oxygen concentration associated with a haematocrit $H$ (this is determined from the vascular problem). Finally, $\lambda_{\text {cell }}$ denotes the assumed constant rate at which cells consume oxygen, with different values for normal and cancerous cells.

The local VEGF profile is calculated in a similar manner, except that cells now act as sources of VEGF and vessels as sinks. If we further assume that VEGF is rapidly eliminated from the vasculature (so that its concentration there is zero) and denote by $\lambda_{V}$ its natural half-life, then $V$ satisfies

$$
0=D_{V} \nabla^{2} V-h_{V} V+\gamma_{\text {cell }}-\lambda_{V} V
$$

In (2.3), $D_{V}$ denotes the assumed constant diffusion coefficient of VEGF and $h_{V}$ the rate at which VEGF crosses vessel walls. Finally, $\gamma_{\text {cell }}$ represents the rate at which sites occupied by cells release their intracellular stores of VEGF to the extracellular environment (this occurs only when the internal levels of VEGF exceed a threshold value). As with $\lambda_{\text {cell }}$ in Eq. (2.2), $\gamma_{\text {cell }}$ differs between normal and cancerous cells.

We note that Eqs. (2.2) and (2.3) for $P$ and $V$ above differ from those used in Refs. 4 and 5 . There the vessels were treated as boundaries and exchange with the vasculature was incorporated as a boundary condition rather than a distributed source term. With a hexagonal network of vessels, this meant that oxygen and VEGF were confined to hexagonal regions of the tissue. The simulations presented below show how the modified equations resolve the physically unrealistic artefact of the earlier simulations and allow the diffusible chemicals to spread more widely through the tissue. As a result of our new treatment of VEGF, we observe spatiotemporal oscillations in the tissue composition when the adaptation of the vasculature is regulated by VEGF (see Fig. 5).

\subsection{The cellular level}

The dynamics of the cell colony is modelled using a two-dimensional cellular automaton, with $N \times N$ automaton elements or cells. ${ }^{39}$ Each element is characterised by a state vector, whose components correspond to features of interest. These include: (i) occupation status (whether an element is occupied by a normal cell, a cancer cell, an empty space or a vessel), (ii) cell status (whether the cell is in a proliferative or quiescent state), (iii) the local oxygen concentration, and (iv) the intracellular levels of VEGF, p53 and cell-cycle proteins (see Sec. 2.4). The state vector evolves according to prescribed local rules that update a given element on the basis of its own state and those of its neighbours at the previous time step. ${ }^{2}$ These rules were inspired by generic features of tumour growth, such as the ability of cancer cells to elude the control mechanisms which maintain stasis in normal tissues. They can also manipulate their local environment, providing themselves with better conditions for growth and, eventually, for invasion of the host organism. ${ }^{24}$ 
Additionally, we endow the cancer cells with the ability to survive exposure to hypoxia for longer than their normal counterparts.

On each timestep the elements of the cellular automaton are updated sequentially. If a particular element is occupied by a cell, we update its internal dynamics (cell cycle proteins, VEGF and p53 levels - see Sec. 2.4). If a cell is ready to divide, the daughter cell is placed in the adjacent cell with the largest oxygen concentration. If there is no empty site, the cell fails to divide and dies. The mechanism for cell death or apoptosis differs between normal and cancer cells. In normal cells, if the intracellular level of p53 exceeds a threshold value (which depends on the cellular composition of its nearest neighbours), then the cell dies. By contrast, under low oxygen, cancer cells become quiescent, suspending most cell functions, including proliferation. On entering this state, a clock is started. It is incremented by unit steps for each timestep that the cell remains quiescent. If the clock reaches a threshold value then the cell dies. However, if the oxygen level increases above the threshold for cancer cell quiescence then the cell recommences cycling and its clock is reset to zero.

\subsection{The subcellular level}

We formulate systems of ordinary differential equations (ODEs) to describe the evolution of the chemicals that control the subcellular processes of interest. These include progress through the cell cycle, VEGF and p53 expression.

The cell cycle is regulated by complex interactions between a large number of proteins, the key components being the families of cyclin-dependent kinases (CDKs) and the cyclins. The activity of cyc-CDK complexes is low during G1 and becomes high after transition. In addition, activities of anaphase protein complex (APC) and the protein Cdh1 are high in G1 but become low after the G1/S transition. For simplicity, we base our work on the model of Tyson and Novak, ${ }^{54}$ which captures the essential features of the cell cycle and can be written in the following form:

$$
\begin{aligned}
\frac{d x}{d t} & =\frac{\left(k_{3}^{\prime}+k_{3}^{\prime \prime} A\right)(1-x)}{J_{3}+1-x}-\frac{k_{4} m y x}{J_{4}+x}, \\
\frac{d y}{d t} & =k_{1}-\left(k_{2}^{\prime}+k_{2}^{\prime \prime} x\right) y, \\
\frac{d m}{d t} & =\mu m\left(1-\frac{m}{m_{*}}\right),
\end{aligned}
$$

where $x \equiv[\mathrm{Cdh} 1]$ is the concentration of active Cdh1/APC complexes, $y \equiv[\mathrm{Cyc}]$ is the concentration of cyclin-CDK complexes and $m$ is the mass of the cell. The parameters $k_{i}(i=1,2,3,4)$ and $J_{i}(i=3,4)$ are positive constants and $A$ represents a generic activator. In Eq. (2.6), $\mu$ is the cell growth rate and $m_{*}$ is the mass of an adult cell.

Equations (2.4)-(2.6) can exhibit mono- and bi-stability, with the cell mass $m$ as a bifurcation parameter. For low values of $m$ there is a single stable steady 
state with a high value of $x$ and a low value of $y$ - this corresponds to G1. As $m$ increases, the system becomes bistable and a new stable steady state characterised by a high value of $y$ and a low value of $x$ emerges. For a critical value of $m$ the latter becomes the only stable steady state and the system switches to this state, corresponding to the $\mathrm{S}$ phase. After the cell divides, $m$ decreases, and the system is re-set to the "G1 phase steady state".

Guided by experimental results presented in Ref. 22 and the hypothesis that under hypoxia expression of the regulatory protein p27 increases,${ }^{27}$ we generalise Eqs. (2.4)-(2.6) and study the (non-dimensionalised) model (see Ref. 3 for full details):

$$
\begin{aligned}
\frac{d x}{d \tau} & =\frac{\left(1+b_{3} u\right)(1-x)}{J_{3}+1-x}-\frac{b_{4} m x y}{J_{4}+x} \\
\frac{d y}{d \tau} & =a_{4}-\left(a_{1}+a_{2} x+a_{3} z\right) y \\
\frac{d m}{d \tau} & =\eta m\left(1-\frac{m}{m_{*}}\right) \\
\frac{d z}{d \tau} & =\chi(m)-c_{2} \frac{P}{B+P} z \\
\frac{d u}{d \tau} & =d_{1}-\left(d_{2}+d_{1} y\right) u
\end{aligned}
$$

where now $P$ is the oxygen tension (see Eq. (2.2)), $z$ is the p27 concentration and $u$ is the concentration of phosphorylated retinoblastoma (RB). In Eqs. (2.8)-(2.11) we assume that normal and cancer cells differ only in the rates at which they produce p27. For normal cells, p27 expression is regulated by cell size and we fix $\chi(m)=c_{1}^{n}\left(1-\frac{m}{m_{*}}\right)$. For cancerous cells, this size-regulation is lost and $\chi(m)=c_{1}^{t}$. To account for the lower levels of p27 observed in cancer cells compared to normal cells ${ }^{44}$ we further assume that the maximum rate of p27 synthesis in normal cells exceeds that in cancer cells (i.e. $c_{1}^{n}>c_{1}^{t}$ ).

Equation (2.10) renders explicit how our cell cycle model is coupled to the tissue level by the oxygen concentration. As we now explain, the other intracellular processes of interest (i.e. VEGF production and apoptosis) are also influenced by $P$. In normal and cancer cells hypoxia stimulates the expression of VEGF and the protein p53. In normal cells p53 stimulates apoptosis and may inhibit VEGF production. ${ }^{49}$ In cancer cells mutations in p53 are common and the protein may upregulate VEGF production and/or prevent apoptosis.

We denote by $p$ and $q$ the concentrations of p53 and VEGF within a given cell and assume that their evolution is modulated by $P$ in the following manner:

$$
\begin{aligned}
& \frac{d p}{d t}=k_{7}-\frac{k_{7}^{\prime} P}{C+P} p, \\
& \frac{d q}{d t}=\xi(p, q)-\frac{k_{8}^{\prime} P}{D+P} q,
\end{aligned}
$$


where

$$
\xi(p, q)= \begin{cases}k_{8}-k_{8}^{n} \frac{p q}{J_{s}^{n}+q} & \text { for normal cells } \\ k_{8}+k_{8}^{t} \frac{p q}{J_{s}^{t}+q} & \text { for cancer cells }\end{cases}
$$

and all parameters are positive. Thus VEGF expression increases under hypoxia. Further p53 increases VEGF production in cancer cells and decreases it in normal cells. The intracellular levels of $p$ and $q$ are assigned to the automaton state vector (see Sec. 2.3).

\subsection{Numerical algorithm}

When performing simulations, the following algorithm is used on each timestep:

(1) Update the vascular network using the structural adaptation rules;

(2) Calculate the blood flow and haematocrit at all points within the vascular network;

(3) Update the oxygen and VEGF profiles by solving the relevant boundary value problems;

(4) Update the elements of the cellular automaton, and the intracellular variables associated with each non-empty element according to whether it is occupied by a normal or cancerous cell.

Further details and a discussion of the parameter values can be found in Ref. 5 .

\subsection{Simulations}

Figures 2-4 show how the spatial composition of the tissue changes over time while Fig. 5 summarises the dynamics of the tumour cells. As stated in Sec. 2.1 there is a single inlet (outlet) to the vasculature located in the bottom left (top right) hand corner of the tissue. As a result, the incoming haematocrit becomes diluted as it passes through the hexagonal lattice. This creates a heterogeneous oxygen distribution across the domain, with oxygen levels being highest near the inlet and outlet. Over time, the tumour cells proliferate and spread through the tissue towards the oxygen-rich regions. As the number of tumour cells increases, their demand for oxygen outstrips that available from the vasculature, leading to the formation of quiescent cells. These cells produce VEGF which diffuses through the tissue (see Eq. (2.3) and Figs. 3 and 4), stimulating vessel adaptation (see Eq. (2.1)) and biasing blood flow towards low oxygen regions. If the vasculature does not adapt to the VEGF stimulus quickly enough then the quiescent cells die. VEGF levels also decline and blood flow to the remaining tumour cells rises, enabling them to increase in number until their demand for oxygen exceeds that being supplied, and so the cycle repeats, with pronounced oscillations in the number of quiescent cells (see Fig. 5). In order to highlight the key role played by VEGF in creating these oscillations, also presented in Fig. 5 are the results of a simulation which was 

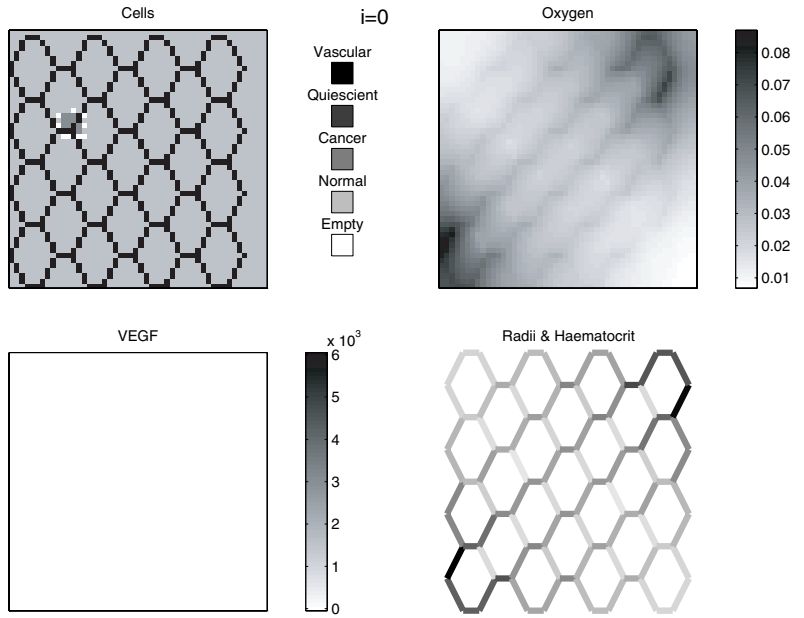

Fig. 2. Series of panels showing the initial spatial distribution of the cells, the oxygen concentration, the VEGF concentration, and the radii and haematocrit associated with each branch of the vascular network, the width of the lines being proportional to the vessel radii and the colour intensity being indicative of the haematocrit (dark = high levels of $H$ ). We note that the seeded tumour is surrounded by a number of empty sites in which it may locate its progeny. From the plots on the right-hand side we observe that the oxygen is distributed nonuniformly across the domain, with the highest levels occurring near the vessel inlet (bottom left corner) and the outlet (top right corner) and corresponding to regions where the haematocrit is largest. Since there are no quiescent cells, there is no VEGF present. Haematocrit and oxygen are maximal (initially all vessels have the same radii) along the diagonal connecting the vessel inlet and outlet.
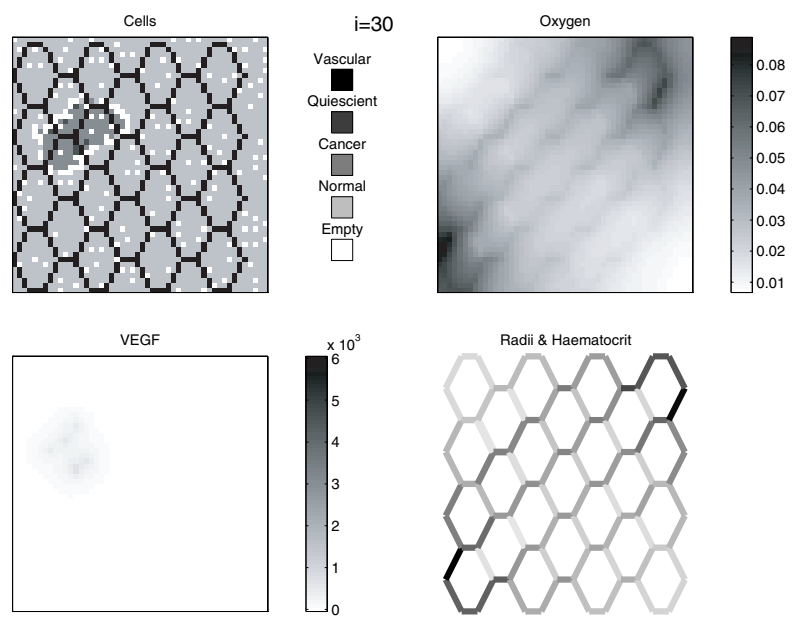

Fig. 3. Series of plots showing how the initial conditions presented in Fig. 2 have developed at $t=30$ (dimensionless time units). The tumour has increased in size and contains regions of quiescence which produce trace amounts of VEGF. The oxygen and vessel profiles remain similar. 

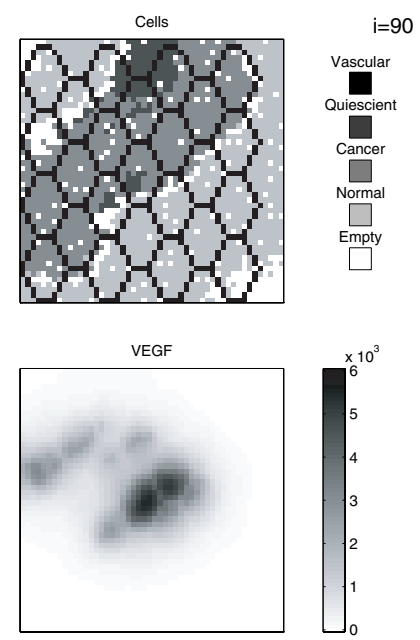
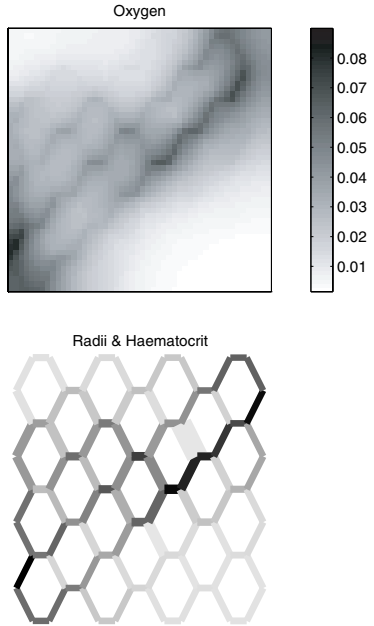

Fig. 4. Series of plots showing how the simulation presented in Figs. 2 and 3 has developed at $t=90$ (dimensionless time units). The tumour continues to expand into the tissue region. There are sufficient numbers of quiescent cells to elicit an angiogenic response, with VEGF localised in a neighbourhood of the quiescent cells (compare the profiles on the left hand side). The profiles on the right hand side show that the VEGF has caused remodelling of the vasculature, with blood flow and oxygen supply (haematocrit) being directed primarily towards the tumour mass.

identical in all aspects except that vascular adaptation was independent of VEGF $\left(k_{m}^{V}=0\right.$ in Eq. (2.1)). In both cases, the tumours grow to similar sizes. However, when vascular adaptation is independent of VEGF, the evolution is monotonic, the oscillations in the cell populations disappear and the number of quiescent cells is consistently much lower. These results are new and show how coupling between the different spatial scales can effect not only the tumour's growth dynamics but also its spatial composition, i.e. the proportion of proliferating and quiescent cells that it contains.

\section{Chemotherapy}

We now investigate how the dynamics of the normal and cancer cells change when a chemotherapeutic drug is introduced. We assume that the drug is continuously administered to the vessels and, hence, that its concentration at the inlet vessel is constant. We calculate the drug concentration $\theta_{\text {vess }}$ within the vascular network in a manner similar to that used to determine the haematocrit, $H$ (see Sec. 2.1). In particular, we assume that the drug is advected with the blood and is partitioned at branch points such that the amount of drug entering the daughter vessels is proportional to their respective flow velocities. As with the oxygen, we view the vessels as distributed sources of drug and assume that, once the drug leaves the vessels, it diffuses through the tissue and is taken up by the normal and healthy cells. 

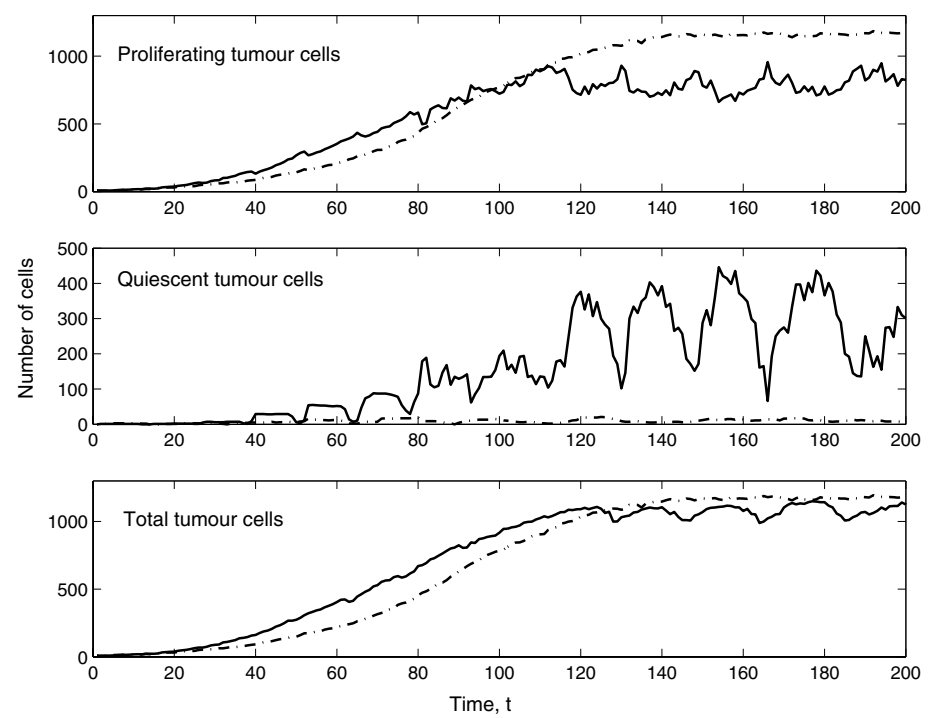

Fig. 5. Series of curves showing how, for the simulations presented in Figs. 2-4, the numbers of proliferating (upper panel), quiescent tumour cells (middle panel) and total number of tumour cells (lower panel) change over time. While there is a fairly steady increase in the number of proliferating cells, the dynamics of the quiescent cells are more complex, initially undergoing oscillations of increasing amplitude. For $t>80$, the tumour is sufficiently large that the quiescent cells are never eliminated: quiescent cells that die are replaced by proliferating cells that become quiescent. The dot-dashed lines show the evolution of a tumour which is identical in all respects except that its vasculature is not regulated by VEGF (so that $k_{m}^{V}=0$ in Eq. (2.1)). While in both cases the tumour reaches a similar equilibrium size, when vascular adaptation is independent of VEGF the oscillations in the cell populations disappear and the number of quiescent cells is much lower.

Guided by Eq. (2.2) and denoting by $\theta$ the drug concentration in the tissue, on each timestep we solve the following diffusion equation, with no-flux boundary conditions, to determine $\theta$ :

$$
0=D_{\theta} \nabla^{2} \theta+h_{\theta}\left(\theta_{\text {vess }}-\theta\right)-\lambda_{\text {drug }} \theta .
$$

In (3.1), $D_{\theta}$ denotes the assumed constant diffusion coefficient of the drug, $h_{\theta}$ the rate at which it is transported across the vessel wall and $\lambda_{\text {drug }}$ the assumed constant rate at which cells absorb the drug (we assume that $\lambda_{\text {drug }}$ is the same for normal and cancerous cells). We remark that natural decay of the drug has been assumed to be dominated by its uptake by the normal and cancer cells (for simplicity, only): it would be straightforward to extend the model to account for drug decay also.

Having determined $\theta_{\text {vess }}$ and $\theta$, it remains to specify the drug's mode of action. Ultimately, we hope to tailor our model to specific drugs according to the stage(s) of the cell cycle at which they act. However, for simplicity, here we assume that the drug works in the following manner. When a cell attempts to divide, if the local drug concentration exceeds a threshold value, then the cell fails to divide and is itself killed. 


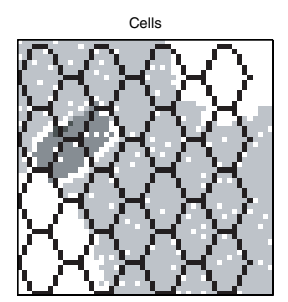

Drug

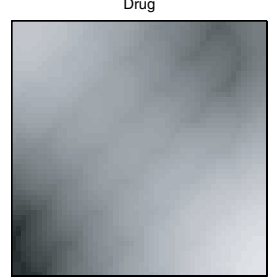

$\mathrm{i}=30$
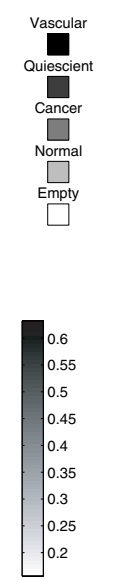

Oxygen

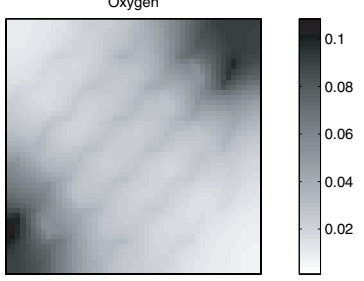

Radii \& Haematocrit

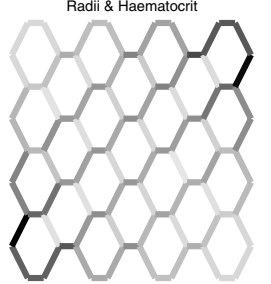

Fig. 6. Series of plots showing how the tissue presented in Fig. 2 responds when an antiproliferative drug is continuously administered through the vasculature. For comparison with Fig. 3, the profiles are plotted at time $t=30$ (dimensionless time units), with the drug distribution replacing the VEGF profile. The drug levels are highest near the inlet and outlet to the vasculature and this causes extensive killing of normal cells, leaving the tumour relatively free of damage.

With these changes we are able to use our model to test the response of our virtual tumour to different drugs. Repeated simulations suggest that, when the drug is used, three qualitatively different types of behaviour emerge: the drug is ineffective and the tumour continues to colonise the tissue (since the dynamics here are similar to those without the drug, no results are presented), the tumour is reduced in size, but not completely eliminated (see Figs. 6-8), or the drug successfully eliminates the tumour (Figs. 9-11).

The simulation presented in Figs. 6-8 corresponds to a case for which the drug fails to eliminate completely the tumour. It reduces the mean size of the tumour at long times and alters its composition so that it comprises predominantly proliferating cells. Further the spatio-temporal oscillations observed in the absence of treatment $\left(h_{\theta}=0\right)$ become more pronounced (see Fig. 12, $h_{\theta}=90$ ) as a result of additional cell death. In more detail, the quiescent tumour cells produce VEGF, stimulating enhanced blood flow and, hence, increased drug delivery. The drug causes the tumour to shrink but, as oxygen consumption is reduced, the number of quiescent cells falls and, in consequence, blood flow and drug delivery decline. The tumour then proliferates into empty regions where the normal cells have been killed by the drug and where oxygen levels are high. The formation of quiescent regions within the tumour mass then stimulates vascular remodelling, redirecting oxygen and drug to these regions (compare Figs. 6-8). The simulation also provides some indication of the degree of damage sustained by the healthy cells. We note that cells located near the inlet and outlet are killed since drug levels are highest there. 

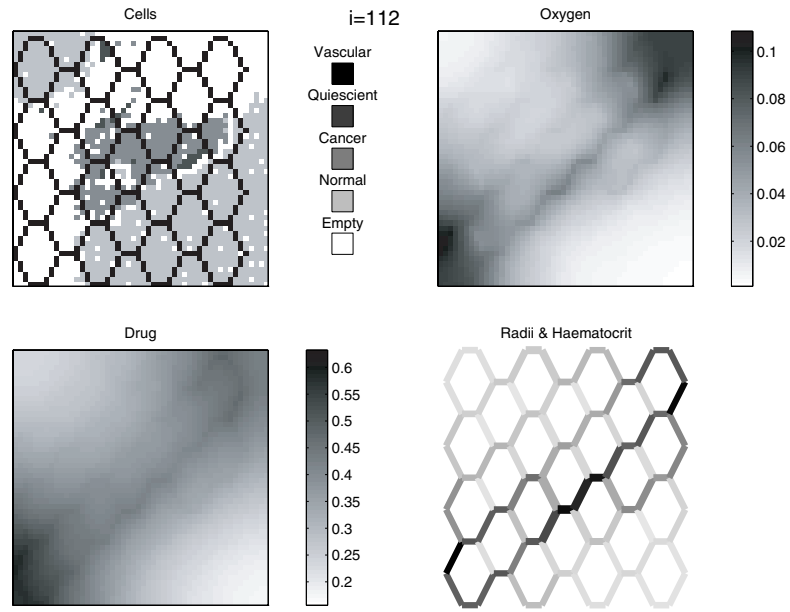

Fig. 7. Series of plots showing how the tissue presented in Figs. 2 and 6 evolves at $t=112$ when an anti-proliferative drug is continuously administered through the vasculature. The quiescent tumour cells produce VEGF, stimulating enhanced blood flow and drug delivery to the tumour region. The drug causes the tumour to shrink, reducing oxygen consumption, the number of quiescent cells and, in consequence, blood flow and drug delivery there. At later times the tumour can repopulate the region and increase in size (see Fig. 8 and the relevant curve in Fig. 12).
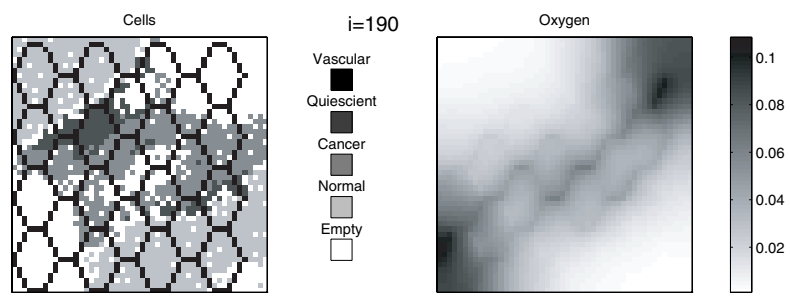

Drug
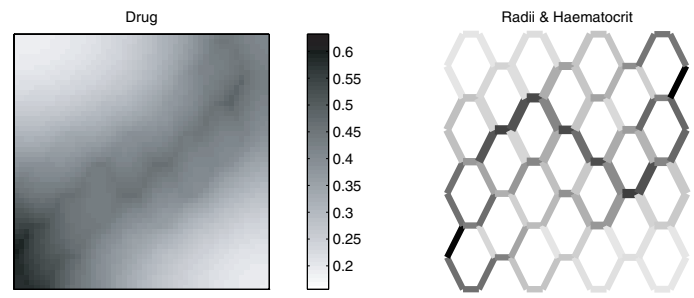

Fig. 8. Series of plots showing how the tissue presented in Figs. 2, 6 and 7 evolves at $t=190$ when an anti-proliferative drug is continuously administered through the vasculature. The tumour is spreading further into the tissue but has a more fragmented and less compact morphology than its untreated counterpart. Remodelling of the vasculature caused by VEGF expression from quiescent cells has led to greater localisation of the oxygen within the tumour region (compare with Fig. 7). 

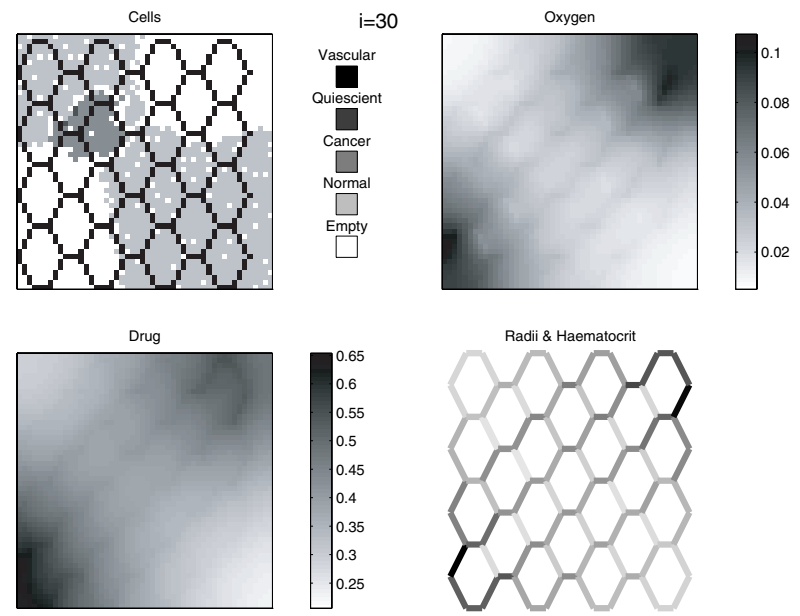

Fig. 9. Series of plots showing how the tissue presented in Fig. 2 evolves at $t=30$ when an antiproliferative drug is continuously administered through the vasculature. The drug is identical to that used to generate Figs. 6-8, except that it crosses the vessel wall more rapidly ( $h_{\theta}$ in Eq. (3.1) increased from $h_{\theta}=90$ to $\left.h_{\theta}=100\right)$. The increase in drug levels causes a marked reduction in the number of tumour cells (compare with Fig. 6) and more extensive damage to the healthy cells.
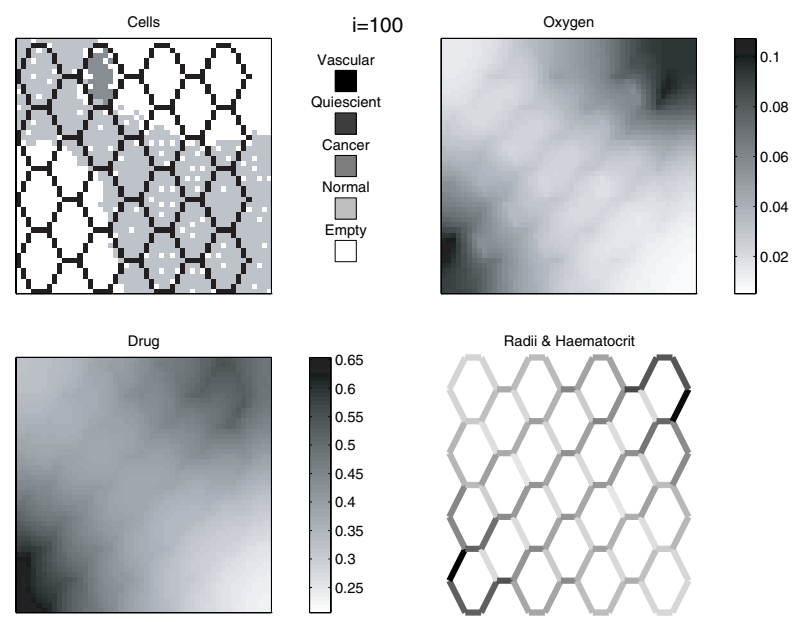

Fig. 10. Series of plots showing how the tissue presented in Figs. 2 and 9 evolves at $t=100$ when an anti-proliferative drug is continuously administered through the vasculature. The tumour is being progressively eliminated.

We remark also that the times used in Figs. 6-8 do not coincide with those used in Figs. 3 and 4: they were chosen to illustrate best the phenomena being described (and similarly in Figs. 9-11).

The simulation presented in Figs. 9-11 shows how the drug may eliminate the tumour. The drug is identical to that used in Figs. 6-8, except that it crosses the 

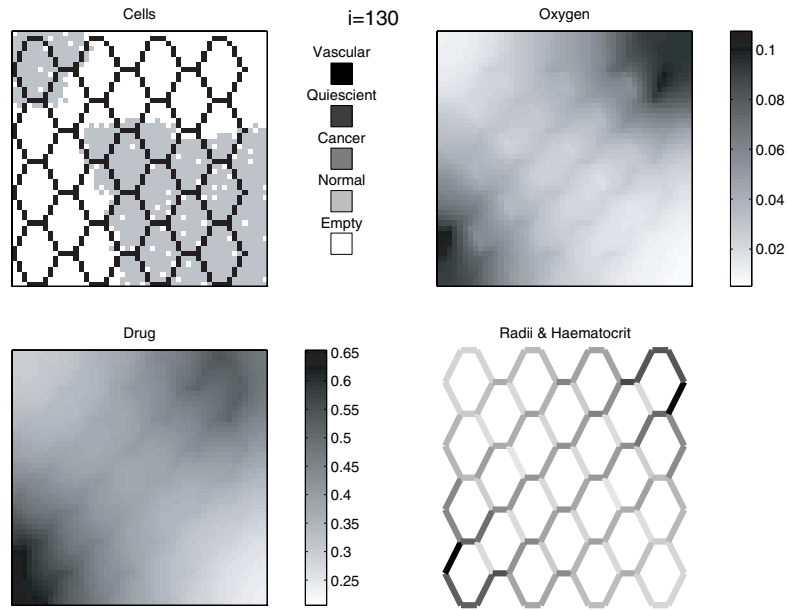

Fig. 11. Series of plots showing how the tissue presented in Figs. 2, 9 and 10 evolves at $t=130$ when an anti-proliferative drug is continuously administered through the vasculature. The tumour has been successfully eliminated, without completely destroying the normal cells.
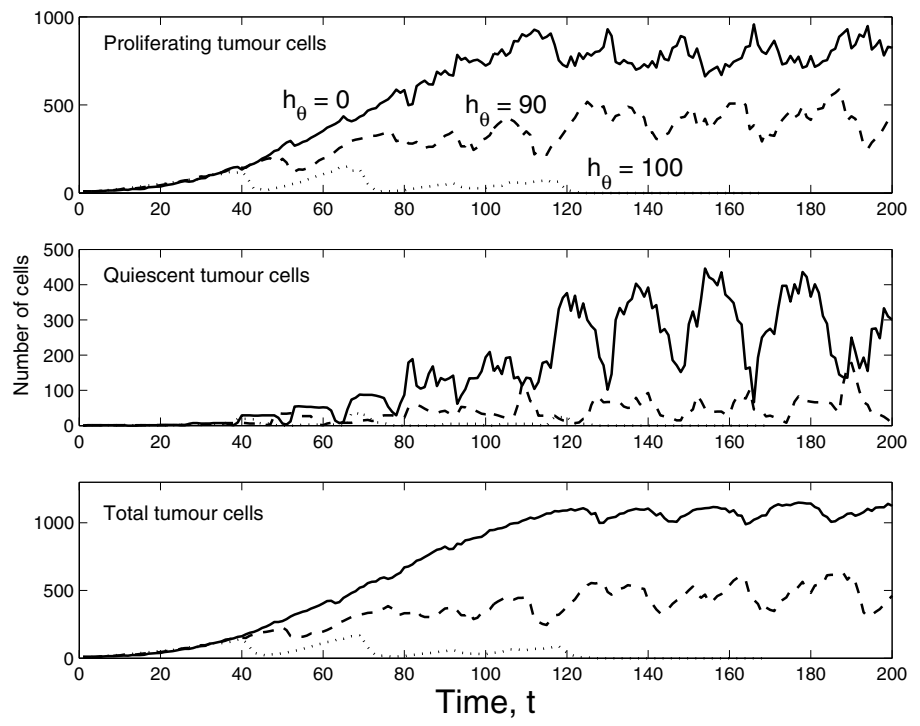

Fig. 12. Series of curves summarising the results of Figs. $2-4\left(h_{\theta}=0\right), 6-8\left(h_{\theta}=90\right)$ and $9-11$ $\left(h_{\theta}=100\right)$. For each simulation, we show how the numbers of proliferating and quiescent tumour cells and the total number of tumour cells evolve over time. Key: $h_{\theta}=0$, solid line; $h_{\theta}=90$, dashed line; $h_{\theta}=100$, dotted line.

vessel walls more quickly $\left(h_{\theta}\right.$ in Eq. (3.1) is increased from $h_{\theta}=90$ in Figs. $6-8$ to $h_{\theta}=100$ in Figs. 9-11). In addition to causing more widespread damage to the normal cells, at early times the increased drug levels also reduce the number of tumour cells (compare Figs. 6 and 9). At later times, drug levels remain high, 
preventing the tumour from spreading through the tissue (compare Figs. 7 and 10) and eventually eliminating it (compare Figs. 8 and 11). We remark that fluctuations in VEGF cause vessel remodelling, fluctuations in the local blood flow and, hence, changes in the oxygen and drug levels. These allow the tumour to enjoy periods of recovery before it is eliminated (see Fig. 12). We also note that while some normal cells are killed by the drug, they are not completely destroyed by the drug when the tumour is eliminated (the drug elicits a stronger response in the tumour cells since they proliferate more rapidly than the normal cells). Thus if treatment were halted at $t=90$ then the healthy cells would eventually repopulate the tissue.

Figure 12 summarises the tumour's dynamics for the three simulations presented in Figs. 2-4 $\left(h_{\theta}=0\right), 6-8\left(h_{\theta}=90\right)$ and 9-11 $\left(h_{\theta}=100\right)$. As $h_{\theta}$ increases, and more drug is delivered, oscillations in the number of proliferating cells become more marked. For sufficiently large values of $h_{\theta}$ (here $h_{\theta} \geq 100$ ) the drug eventually eliminates the tumour.

\section{Conclusions}

We have shown how a multiscale model can be used to study the response of a vascular tumour to continuous infusion with chemotherapy. We have used a modified version of a hybrid cellular automaton model that was originally developed by Alarcón et al. ${ }^{4,5}$ and which incorporates coupling between phenomena occurring on different spatial scales. By treating the vessels as continuous sources (or sinks) of oxygen and drug (or VEGF), physically unrealistic artefacts of the earlier models have now been removed and the diffusible species are able to spread across the entire tissue region. As a result, we observe spatio-temporal oscillations in the tissue composition when vascular adaptation is regulated by VEGF (see Fig. 5), a feature that, to our knowledge, has not been reported previously in similar mathematical models of vascular tumour growth.

McDougall et $a l .{ }^{36}$ used a similar model of vascular adaptation and blood flow to study the distribution of blood-borne chemotherapy. However, they did not consider the cytotoxic effect of the drug on the underlying tissue. By contrast, we predict the cell kill that results when a particular drug is used. Further, since in our model there is coupling between the subcellular, cellular and macro- or vascular scales, we are also able to determine the (indirect) impact that changes in the cellular composition of the tissue have on the vasculature (this being relevant when a standard chemotherapeutic drug is administered) and vice versa (this being relevant when an antiangiogenic drug is used).

Our simulations show that when a drug that targets proliferating cells (tumour and normal) is continuously administered via the vasculature, the outcome depends on the choice of parameter values and takes three possible forms: the tumour is successfully eliminated by the drug; the tumour's invasion of the healthy tissue continues; or, the tumour grows to a finite size, with more pronounced spatiotemporal oscillations in its composition. 
While the results we obtain are qualitatively realistic, there are many avenues for further investigation. These stem, to a large extent, from the fact that the submodels we have employed are rather simplistic. Since the framework we present is general (see Fig. 1), there is considerable scope for incorporating more realistic submodels and establishing conditions under which the more complex models yield different qualitative behaviour. This is a key issue facing researchers developing multiscale models. ${ }^{10}$ For example, we could implement a more detailed cell cycle model and use it to study the impact of drugs that act at one or more of the phases of the cell cycle or those which target the tumour's vasculature. Conversely, we could introduce a simpler model in which decisions about cell division are based solely on a cell's local environment. While these investigations may appear rather theoretical in nature, comparing the results that they generate should provide useful insight into the level of detail that is needed to model accurately the system's response to therapy. In particular, we anticipate that the degree of detail needed will depend on a particular drug's mode of action (e.g. whether it is cell-cycle specific). Equally, following Gatenby et al. ${ }^{23,24,50}$ we could include a more detailed description of cellular metabolism and investigate the role of $\mathrm{pH}$ on tumour invasion. Other important mechanisms that are the subject of ongoing work include a more detailed description of angiogenesis (with explicit treatment of capillary tip formation and tip-to-tip anastomosis), the impact of mechanical effects (e.g. contact inhibition of cell proliferation ${ }^{13,32}$ and the collapse of immature vessels under pressure $^{12}$ ) and cell movement. ${ }^{6}$

We could also adapt the model to compare the tumour/tissue response to different drug delivery protocols, including combination treatments and possibly involving novel types of gene therapy that target hypoxic regions ${ }^{41}$ and allowing for tumour cell heterogeneity, with some subclonal populations being resistant to therapy. The simulations we presented focused on the tumour's response to continuous infusion with a chemotherapeutic drug but also highlighted the extensive damage to normal cells that is an inevitable consequence of such highly toxic compounds. In practice these side-effects mean that the drugs are administered as a series of boluses, rather than by continuous infusion. Our model could easily be adapted to study this more realistic situation. Other interesting directions for future research involve using kinetic theory and statistical mechanics in place of cellular automata to simulate cell dynamics ${ }^{11}$ and incorporating concepts from evolutionary game theory to study carcinogenesis. ${ }^{26}$ In the latter case random genetic mutations introduce heterogeneity into the cell population, the fitness of the different subpopulations being influenced by intra- and extra-cellular factors. As a result, we could investigate how tumours are initiated and whether environmental conditions select for particular genetic mutations.

One weakness of our multiscale model that stems from its complexity is the reliance on numerical simulations to obtain solutions. This may limit the applicability of the model. For example, it may be infeasible to run large numbers of simulations on large spatial domains. However, by validating the model against detailed 
spatio-temporal data from, for example, mouse chamber assays, ${ }^{14}$ it should be possible to extract from the simulations averaged time activity curves, similar to those presented in Figs. 5 and 12, showing how the numbers of tumour cells (proliferating and quiescent) and normal cells, together with the mean vascular density, evolve over time. These curves could then be fit to lumped parameter models, similar to those being developed by Arakelyan et al. ${ }^{7}$ before the (indirectly validated) ODE models are used to study less detailed data from large scale clinical trials. In doing this, it will of course be necessary to specialise the model to describe specific tumour types ${ }^{47,52}$ and particular drugs. ${ }^{15,33,42}$ This is the subject of ongoing work.

One of the major challenges facing researchers in the Life Sciences is understanding how processes interact on different length and time scales. Verbal reasoning is not sufficient to compute the outcome of such complex interactions and we must therefore use computational modelling. However, if we were to express every known reaction computationally, we would arrive at a model that was computationally intractable and impossible to parametrise. Therefore we must use coarse-graining and herein lies the mathematical challenge. For example, in the modelling described in this paper we used for the cell cycle a very simple model that captured the essence of certain aspects of the cell cycle (which has been modelled using up to 60 equations). Can we make similar simplifications for other biochemical networks? Can we be certain that we have not lost key information by making this simplification? That is, how robust are the subsequent models? Is it possible that we could describe the framework we presented at a continuum level which would capture the key features of this model yet allow us more easily to scale up to three spatial dimensions and to larger, more realistic, sizes of tissue? It may well be that we need a suite of models depending on what question that we can answer.

Although there have been some attempts to tackle aspects of the issues mentioned above, such as using Boolean switches to understand complex biochemical networks (instead of the more complicated modelling approach of using coupled ordinary differential equations), there has been no systematic approach and these are all open questions that will stimulate theoreticians and experimentalists for many years to come.

\section{Acknowledgments}

T.A. thanks the Engineering and Physical Sciences Research Council for financial support under grant GR/509067. H.M.B. thanks the EPSRC for funding as an Advanced Research Fellow. This work was supported in part by NIH grant CA 113004. The authors wish to acknowledge the support provided by the funders of the Integrative Biology project: The EPSRC (ref no: GR/S72023/01) and IBM.

\section{References}

1. J. A. Adam, A mathematical model of tumour growth. II Effects of geometry and spatial uniformity on stability, Math. Biosci. 86 (1987) 183-211. 
2. T. Alarcón, H. M. Byrne and P. K. Maini, A cellular automaton model for tumour growth in inhomogeneous environment, J. Theor. Biol. 225 (2003) 257-274.

3. T. Alarcón, H. M. Byrne and P. K. Maini, A mathematical model of the effects of hypoxia on the cell-cycle of normal and cancer cells, J. Theor. Biol. 229 (2003) 395-411.

4. T. Alarcón, H. M. Byrne and P. K. Maini, Towards whole-organ modelling of tumour growth, Prog. Biophys. Mol. Biol. 85 (2004) 451-472.

5. T. Alarcón, H. M. Byrne and P. K. Maini, A multiple scale model for tumour growth, Multiscale Model Sim. 3 (2005) 440-475.

6. A. R. Anderson, A hybrid mathematical model of solid tumour invasion: The importance of cell adhesion, Math. Med. Biol. 22 (2005) 163-186.

7. L. Arakelyan, Y. Merbl and Z. Agur, Vessel maturation effects on tumour growth: Validation of a computer model in implanted human ovarian carcinoma spheroids, Eur. J. Cancer 41 (2005) 159-167.

8. R. P. Araujo and D. L. S. McElwain, A history of the study of solid tumor growth: The contribution of mathematical modelling, Bull. Math. Biol. 66 (2004) 1039-1091.

9. B. Basse, B. C. Baguley, E. S. Marshall, G. C. Wake and D. J. Wall, Modelling the flow cytometric data obtained from unperturbed human tumour cell lines: Parameter fitting and comparison, Bull. Math. Biol. 67 (2005) 815-830.

10. N. Bellomo and P. K. Maini, Preface and Special issue on "Multiscale Cancer Modelling - A New Frontier in Applied Mathematics", Math. Mod. Meth. Appl. Sci. 15 (2005) iii-vi; 1619-1794.

11. A. Bellouquid and M. Delitala, Mathematical methods and tools of kinetic theory towards modelling complex biological systems, Math. Mod. Meth. Appl. Sci. 15 (2005) 1639-1666.

12. Y. Boucher and R. K. Jain, Microvascular pressure is the principal driving force for interstitial hypertension in solid tumours: Implications for vessel collapse, Cancer Res. 52 (1992) 5110-5114.

13. A. Brú, S. Albertos, J. Luis Subiza, J. L. García-Asenjo and I. Brú, The universal dynamics of tumor growth, Biophys. J. 85 (2003) 2948-2961.

14. M. Dellian, B. P. Witwer, H. A. Salehi, F. Yuan and R. K. Jain, Quantitation and physiological characterization of angiogenic vessels in mice: Effect of basic fibroblast growth factor, vascular endothelial growth factor/vascular permeability factor, and host microenvironment, Am. J. Pathol. 149 (1996) 59-71.

15. K. R. Fister and J. C. Panetta, Optimal control applied to cell-cycle-specific cancer chemotherapy, SIAM J. Appl. Math. 60 (2000) 1059-1072.

16. J. Folkman and M. Hochberg, Self-regulation of growth in three dimensions, J. Exp. Med. 138 (1973) 745-753.

17. J. Folkman, Tumour angiogenesis, Adv. Cancer Res. 19 (1974) 331-358.

18. J. Folkman, Fundamental concepts of the angiogenic process, Curr. Mol. Med. 3 (2003) 643-651.

19. J. Folkman, Antiangiogenic activity of a matrix protein, Cancer Biol. Ther. 2 (2003) $53-54$.

20. L. M. Franks and N. Teich, Introduction to the Cellular and Molecular Biology of Cancer (Oxford, 1988).

21. Y. C. Fung, Biomechanics (Springer, 1993).

22. L. B. Gardner, Q. Li, M. S. Parks, W. M. Flanagan, G. L. Semenza and C. V. Dang, Hypoxia inhibits $\mathrm{G}_{1} / \mathrm{S}$ transition through regulation of $\mathrm{p} 27$ expression, J. Biol. Chem. 276 (2001) 7919-7926. 
23. R. A. Gatenby and E. T. Gawlinski, A reaction-diffusion model of cancer invasion, Cancer Res. 56 (1996) 5745-5753.

24. R. A. Gatenby and E. T. Gawlinski, The glycolytic phenotype in carcinogenesis and tumor invasion: Insights through mathematical models, Cancer Res. 63 (2003) 38473854.

25. R. A. Gatenby and P. K. Maini, Mathematical oncology: Cancer summed up, Nature 421 (2003) 321.

26. R. A. Gatenby, T. L. Vincent and R. J. Gillies, Evolutionary dynamics in carcinogenesis, Math. Mod. Meth. Appl. Sci. 15 (2005) 1619-1638.

27. S. L. Green, R. A. Freiberg and A. Giaccia, p21 ${ }^{\mathrm{Cip} 1}$ and $\mathrm{p} 27^{\mathrm{Kip} 1}$ regulate cell cycle reentry after hypoxic stress but are not necessary for hypoxia-induced arrest, Mol. Cell. Biol. 21 (2001) 1196-1206.

28. H. P. Greenspan, Models for the growth of a solid tumour by diffusion, Stud. Appl. Math. 52 (1972) 317-340.

29. H. P. Greenspan, On the growth and stability of cell cultures and solid tumours, $J$. Theor. Biol. 56 (1976) 229-242.

30. K. Groebe and W. Mueller-Klieser, Distributions of oxygen, nutrient and metabolic waste concentration in multicellular spheroids and their dependence on spheroid parameters, Eur. Biophys. J. 19 (1996) 169-181.

31. K. Groebe and W. Mueller-Klieser, On the relation between size of necrosis and diameter of tumour spheroids, Int. J. Radiat. Oncol. 34 (1996) 395-401.

32. G. Helmlinger, P. A. Netti, H. C. Lichtenbeld, R. J. Melder and R. K. Jain, Solid stress inhibits the growth of multicellular tumour spheroids, Nature Biotech. 15 (1997) 778783.

33. T. L. Jackson, Intracellular accumulation and mechanism of action of doxorubicin in a spatio-temporal tumor model, J. Theor. Biol. 220 (2003) 201-213.

34. T. L. Jackson and H. M. Byrne, A mathematical model to study the effects of drug resistance and vasculature on the response of solid tumors to chemotherapy, Math. Biosci. 164 (2000) 17-38.

35. T. L. Jackson, S. R. Lubkin and J. D. Murray, Theoretical analysis of conjugate localization in two-step cancer chemotherapy, J. Math. Biol. 39 (1999) 353-376.

36. S. R. McDougall, A. R. A. Anderson, M. A. J. Chaplain and J. A. Sherratt, Mathematical modelling of flow through vascular networks: Implications for tumour-induced angiogenesis and chemotherapy strategies, Bull. Math. Biol. 64 (2002) 673-702.

37. D. L. S. McElwain and L. E. Morris, Apoptosis as a volume loss mechanism in mathematical models of solid tumour growth, Math. Biosci. 39 (1978) 147-157.

38. N. Mantzaris, S. Webb and H. G. Othmer, Mathematical modeling of tumor-induced angiogenesis, J. Math. Biol. 95 (2004) 111-187.

39. J. Moreira and A. Deutsch, Cellular automaton models of tumor development: A critical review, Adv. Complex Syst. 5 (2002) 247-267.

40. G. Nestler, H. U. Schulz, J. Tautenhahn, R. Kuhn, S. Kruger, H. Lippert and M. Pross, Effects of the angiogenesis inhibitor angiostatin on the growth of CC531 colon carcinoma cells in vitro and in a laparoscopic animal model of peritoneal carcinomatosis, Int. J. Colorectal. Dis. 5 (2005) 1-7.

41. M. R. Owen, H. M. Byrne and C. E. Lewis, Mathematical modelling of the use of macrophages as vehicles for drug delivery to hypoxic tumour sites, J. Theor. Biol. 226 (2004) 377-391.

42. J. C. Panetta, A mathematical model of breast and ovarian cancer treated with paclitaxel, Math. Biosci. 146 (1997) 89-113. 
43. A. A. Patel, E. T. Gawlinsky, S. K. Lemieux and R. A. Gatenby, Cellular automaton model of early tumour growth and invasion: The effects of native tissue vascularity and increased anaerobic tumour metabolism, J. Theor. Biol. 213 (2001) 315-331.

44. J. Philipp-Staheli, S. R. Payne and C. J. Kemp, p27(Kip1): Regulation and function of haploinsufficient tumour suppressor and its misregulation in cancer, Exp. Cell. Res. 264 (2001) 148-168.

45. L. Preziosi, Cancer Modelling and Simulation (CRC Press, 2003).

46. A. R. Pries, T. W. Secomb and P. Gaehtgens, Structural adaptation and stability of microvascular networks: Theory and simulations, Am. J. Physiol. 275 (1998) H349H360.

47. B. Ribba, K. Marron, Z. Agur, T. Alarcón and P. K. Maini, A mathematical model of doxorubicin treatment efficacy for non-Hodgkin's lymphoma: Investigation of the current protocol through theoretical modelling results, Bull. Math. Biol. 67 (2005) 79-99.

48. T. Roose, S. J. Chapman and P. K. Maini, Mathematical models of avascular tumour growth, submitted.

49. J. A. Royds, S. K. Dower, E. E. Qwarstrom and C. E. Lewis, Resonse of tumour cells to hypoxia: role of p53 and NF $\kappa \beta$, J. Clin. Path. Mol. Pathol. 51 (1998) 55-61.

50. K. Smallbone, D. J. Gavaghan, R. A. Gatenby and P. K. Maini, The role of acidity in solid tumour growth and invasion, J. Theor. Biol. 235 (2005) 476-484.

51. A. Stephanou, S. R. McDougall, A. R. A Anderson and M. A. J. Chaplain, Mathematical modelling of the influence of blood rheological properties upon adaptive tumour-induced angiogenesis, J. Math. Comp. Mod., submitted.

52. K. R. Swanson, C. Bridge, J. D. Murray and E. C. Alvord, Virtual and real brain tumors: Using mathematical modeling to quantify glioma growth and invasion, $J$. Neur. Sci. 216 (2003) 1-10.

53. G. M. Tozer, C. Kanthou, C. S. Parkins and S. A. Hill, The biology of the combretastatins as tumour vascular targeting agents, Int. J. Exp. Pathol. 83 (2002) 21-38.

54. J. J. Tyson and B. Novak, Regulation of the eukaryotic cell-cycle: Molecular anatagonism, hysteresis, and irreversible transitions, J. Theor. Biol. 210 (2001) 249-263. 